\title{
Improving the stiffness of multilayer 3D woven composites by the integration of shape memory alloys (SMAs) into structures
}

\begin{abstract}
Shape memory alloys (SMAs) are capable of shape-retaining and stress generation when activated. SMA wires are embedded in laminated composites for improving the properties of the composites. Laminated composites have low through-the-thickness properties and poor delamination resistance. 3D composites are well known for having higher through-thethickness properties. In 3D woven composites, a set of yarn is in through-the-thickness direction that improves through-the- thickness properties and provides resistance to delamination of layers. As in multilayer 3D woven structures, yarns are distributed from inplane to through-the-thickness direction, so in-plane properties are reduced with the same number of yarns compared to 2D laminated composites. In this research, SMA wires are embedded into different types of 3D woven structures for utilising stress generation property of SMA wires for improving in-plane properties, specifically stiffness of the composites. Three types of 3D orthogonal interlocking composites: layer-to-layer, through-the-thickness, and modified multilayer interlock structures are fabricated with and without SMA wires. From the tensile test, results show that embedding SMA wires into structures significantly improves the stiffness of the structures due to the stress-induced martensite phase of SMA wire when subjected to load. When these SMA wires are activated, stresses are generated by SMA wires due to phase transformation from martensite to austenite that further gives remarkable higher values of stiffness. This results in a composite structure that has higher inplane properties due to embedded SMA wire and through-the-thickness properties due to 3D structure of composite reinforcement. The interlocking pattern in the through-the-thickness direction of 3D structures was also found to have an effect on the extent of the improvement in stiffness.
\end{abstract}

Keyword: Austenite; Martensite; Shape Memory Alloys (SMAs); Stiffness; Through-the-thickness properties; 3D woven composite 